\title{
Prevalence of domestic violence in a time of catastrophic disease outbreaks including COVID 19 pandemic: A systematic review protocol
}

Gelila Abraham ( $\sim$ abrahamgelila7@gmail.com )

Jimma University, Institute of Health, Public Health Faculty https://orcid.org/0000-0002-6506-2750

\section{Beshea Gelana}

Jimma University, Institute of Health

Kiddus Yitbarek

Jimma University, Institute of Health

Sudhakar Morankar

Jimma University, Institute of Health

\section{Protocol}

Keywords: Domestic violence, disease outbreak, pandemic, epidemic, prevalence, vulnerable groups

Posted Date: December 8th, 2020

DOI: https://doi.org/10.21203/rs.3.rs-121887/v1

License: (9) This work is licensed under a Creative Commons Attribution 4.0 International License.

Read Full License

Version of Record: A version of this preprint was published at Systematic Reviews on March 17th, 2022.

See the published version at https://doi.org/10.1186/s13643-022-01920-9. 


\section{Abstract \\ Background}

Domestic violence is a public health issue that has a long term and irreversible effect on the victims. There are vulnerable groups like children, women, and elders. The problem becomes worse for these populations in the time of catastrophic events including disease pandemic. However, few attempts have been made to systematically review the prevalence and pattern of domestic violence during these times all over the world.

\section{Methods}

An initial search of PubMed will be followed by CINAHL, Scopus, Google Scholar, Embase, and Proquest Health. Titles and abstracts of studies will be reviewed, and full-text articles will be selected if the inclusion criteria are met. Studies that meet the eligibility criteria will then be assessed by two independent reviewers. Full-text articles will be selected if the inclusion criteria are met. A standardized critical appraisal checklist for studies reporting prevalence data will be used to assess methodological quality and a standardized data extraction tool will be used. The results from the included studies will be analyzed using JBI SUMARI software.

\section{Discussion}

This systematic review will provide a solid evidence on the magnitude of domestic violence of any forms during catastrophic disease outbreaks including the current pandemic, COVID-19.

\section{Systematic review registration number:}

The systematic review protocol registration number in PROSPERO is CRD42020192255.

\section{Background}

Domestic violence is defined broadly as: "all acts of physical, sexual, psychological or economic violence" that may be committed by a family member or intimate partner. It is also named as 'domestic abuse' or 'family violence' (1). Besides, the World Health Organization (WHO) defines violence as: "the intentional use of power or force, threatened or actual, against oneself, another person or against a group or community, which either results in or has a likelihood of resulting in, injury, death, psychological harm, mal-development or deprivation". Based on this definition WHO separates violence into three broad groups, named, self-directed violence; interpersonal violence; and collective violence. Self-directed violence includes suicidal behaviour (i.e., suicidal ideation, plans, attempted suicide, and suicide). Interpersonal violence by itself divides into two categories, i.e., family and intimate partner violence (e.g., 
child abuse, violence by an intimate partner, and abuse of the elderly) and community violence (e.g., youth violence, rape or sexual assault by strangers and violence in institutional settings). Collective violence includes wars and armed conflicts within or between states, genocide, and terrorism (2).

It is well known that violence has probably always been part of the human experience and its impact can be seen, in various forms, in all parts of the world. All types of violence are related to human distress and disastrous. Natural disasters including pandemic might increase the rate of violence both in the short and long-term in a number of ways (3). Domestic violence is the most common form of violence against children, women, and elderly persons. It affects the victims up to the life span, from self-selective to forced suicide and abuse, and is evident, to some degree, in every society in the world. There is a huge body of research showing the lasting effects of domestic violence on victims which includes; mental health problems and chronic illnesses (4). There is also evidence which shows, in one year, approximately 300 million women aged 15-64 are assaulted by an intimate partner: that is every ninth woman in the world, every year (5). There is increasing recognition internationally that intimate partner violence is a common problem with serious health, social and economic consequences for women, their families, and communities (6).

Domestic violence is now viewed as global public health and clinical problem of epidemic proportions. For women and girls, being subjected to violence is associated with: injury, disability, death, induced abortion, low birth weight and prematurity in women's babies, poor sexual health, suicide, depression, anxiety, and harmful alcohol use (7). In the middles of disaster; be it a natural disaster or disease outbreak, the physical and social environments that shape the health and health problems will disrupt. Therefore, the effects of disasters are likely to increase in individuals, families, and communities' vulnerability to violence and effects can have both an immediate and long-term impact on violence (8). Furthermore, due to the scarcity of basic provisions, failure of law enforcement, powerlessness, aggravation caused by a deceitful bureaucracy, and forgotten governments' potentials to help victims, etc. It would be possible that the mental distresses will develop into violence, either self-directed or interpersonal (9). Likewise, studies recently conducted, revealed that interpersonal violence increases during epidemics, like the Ebola virus in West Africa and currently COVID 19 pandemics, globally (10), (11).

There is an evidence showing domestic violence reports often substantially increase after a catastrophic event (12). While the depth and severity of the COVID-19 pandemic are still uncertain, it is clear that households in affected areas will feel some economic shock-with the largest effects among the population of already economically vulnerable. In line with this, women, in particular, may be disproportionately affected by additional unpaid care (caretaking and caregiving) work, which may further decrease the ability to undertake paid work (13). Furthermore, there is a high likelihood of increases in family violence during the COVID-19 pandemic (14). Disruption of family life has a significant effect on children, including poverty (if divorce or separation occurs) and a loss of faith and trust in the association of the family. These squeals not only affect the quality of lives of individuals and societies but also have lasting effects on community order and unity. This could be shown by increased 
interpersonal violence during crises and times of unrest like pandemics (15). However, there are limited rigorous studies estimated an increase in reporting of violence during or post-pandemic though media reports and anecdotal evidence are widespread (16).

Violence and its impact could be preventable in the same way public health efforts have prevented, and reduced pregnancy-related complications, infectious diseases, and many other injuries all over the world. However, there are obstacles that hinder the system not to be effective, including a lack of accurate data on the magnitude and its determinants (17). Therefore, given the alarming increase of natural disasters including pandemics during the recent decades, it is time to design and conduct more methodological sound studies (18)'(19).

A preliminary search of PROSPERO, MEDLINE, the Cochrane Database of Systematic Reviews, and the JBI Database of Systematic Reviews and Implementation Reports was conducted and no current or underway systematic reviews on the topic were identified.

\section{Methods}

The development of this protocol reasonably followed the preferred reporting item for systematic reviews and meta-analyses extension for a protocol (PRISMA-P) guidelines (Supplementary file 1).

\section{Review Objective}

The aim of this review is to synthesize the best available evidence on the prevalence of domestic violence during catastrophic disease outbreaks including COVID 19 pandemic.

\section{Study Design}

The proposed systematic review will be conducted in accordance with the Joanna Briggs Institute methodology for systematic reviews of prevalence and incidence and the Preferred Reporting Items for Systematic Reviews and Meta-Analyses (PRISMA) (20)(21).

\section{Inclusion criteria}

\section{Participants}

The review will consider studies that include children, adolescents, women, elders and any vulnerable groups with or without co-existing health problems.

\section{Condition}


This review will consider studies that report on the prevalence of domestic violence in one or more forms; physical, psychological, and sexual during catastrophic health events (including pandemics and epidemics).

\section{Context}

This review will consider studies that report on the prevalence of domestic violence in the community which is conducted elsewhere in the globe.

\section{Types of studies}

The review will consider observational studies and particularly population-based cross-sectional study. Other study designs that provide indications of the prevalence of domestic violence during catastrophic health events will be considered for inclusion.

Studies published in the English language will be included, while we will not have a restriction to the sample size of the studies. Studies published after 2010 G.C. will be included in the review to capture recent trends in the prevalence of domestic violence during disease epidemics and pandemics.

\section{Search strategy}

The search strategy will aim to locate both published and unpublished studies. An initial limited search of MEDLINE and CINAHL will be undertaken to identify articles on the topic. The text words contained in the titles and abstracts of relevant articles, and the index terms used to describe the articles will be used to develop a full search strategy for report the name of the relevant database (Table 1). The search strategy, including all identified keywords and index terms, will be adapted for each included information source. Searching for additional studies from grey literature from government departments, international agencies, and academic institution repositories will be conducted using similar keywords from the search strings. The reference list of all studies selected for critical appraisal will be screened for additional studies.

Table 1: Search strategy of PubMed database 
MEDLINE (PubMed). Date searched: 13 October 2020

Results retrieved: 8194

(((((("epidemiology"[Subheading] OR "epidemiology"[All Fields] OR "prevalence"[All Fields] OR "prevalence"[MeSH Terms]) AND ("domestic violence"[MeSH Terms] OR ("domestic"[All Fields] AND "violence"[All Fields]) OR "domestic violence"[All Fields])) OR "violence"[MeSH Terms]) OR "physical abuse"[MeSH Terms]) AND "sex offenses"[MeSH Terms]) OR ("disease outbreaks"[MeSH Terms] OR ("disease"[All Fields] AND "outbreaks"[All Fields]) OR "disease outbreaks"[All Fields] OR ("disease"[All Fields] AND "outbreak"[All Fields]) OR "disease outbreak"[All Fields])) OR "epidemics"[MeSH Terms]) AND "pandemics"[MeSH Terms]

\section{Information sources}

The databases to be searched include: PubMed, Scopus, Google Scholar, Embase, Web of Science, survey reports, conference papers, dissertations, bulletins and fact sheets. The trial registers to be searched include: Cochrane Central Register of Controlled Trials and the search for unpublished studies will include survey reports.

\section{Study selection}

Following the search, all identified citations will be collated and uploaded into Mendeley v1.19.6 (Mendeley Ltd., Elsevier, Netherlands) and duplicates removed. Titles and abstracts will then be screened by two independent reviewers for assessment against the inclusion criteria for the review. Potentially relevant studies will be retrieved in full and their citation details imported into the Joanna Briggs Institute System for the Unified Management, Assessment and Review of Information (JBI SUMARI) (22). The full text of selected citations will be assessed in detail against the inclusion criteria by two independent reviewers. Reasons for exclusion of full text studies that do not meet the inclusion criteria will be recorded and reported in the systematic review. Any disagreements that arise between the reviewers at each stage of the study selection process will be resolved through discussion, or with a third reviewer. The results of the search will be reported in full in the final systematic review and presented in a Preferred Reporting Items for Systematic Reviews and Meta-analyses (PRISMA) flow diagram (23).

\section{Assessment of methodological quality}

Eligible studies will be critically appraised by two independent reviewers for methodological quality using standardized critical appraisal instruments for prevalence studies in JBI SUMARI (20) (Table 2). Authors of papers will be contacted to request missing or additional data for clarification, where required. Any disagreements that arise will be resolved through discussion, or with a third reviewer. The results of critical appraisal will be reported in narrative form and in a table. All studies that meet the inclusion criteria, regardless of their methodological quality, will undergo data extraction and synthesis (where possible). 


\begin{tabular}{|c|c|c|c|}
\hline $\begin{array}{l}\text { Study } \\
\text { design }\end{array}$ & Tool & Domains & Overall risk of bias judgement \\
\hline $\begin{array}{l}\text { Cross- } \\
\text { sectional } \\
\text { studies }\end{array}$ & $\begin{array}{l}\text { JBI Critical } \\
\text { Appraisal } \\
\text { Checklist for } \\
\text { studies reporting } \\
\text { Prevalence Data }\end{array}$ & $\begin{array}{l}\text { 1. Was the sample frame } \\
\text { appropriate to address } \\
\text { the target population? } \\
\text { 2. Were study } \\
\text { participants sampled in } \\
\text { an appropriate way? } \\
\text { 3. Was the sample size } \\
\text { adequate? } \\
\text { 4. Were the study } \\
\text { subjects and the setting } \\
\text { described in detail? } \\
\text { 5. Was the data analysis } \\
\text { conducted with sufficient } \\
\text { coverage of the identified } \\
\text { sample? } \\
\text { 6. Were valid methods } \\
\text { used for the } \\
\text { identification of the } \\
\text { condition? } \\
\text { 7. Was the condition } \\
\text { measure in a standard, } \\
\text { reliable way for all } \\
\text { participants? } \\
\text { 8. Was there appropriate } \\
\text { statistical analysis? } \\
\text { 9. Was the response rate } \\
\text { adequate, and if not, was } \\
\text { the low response rate } \\
\text { managed appropriately? }\end{array}$ & $\begin{array}{l}\text { An item would be scored "0" if it was } \\
\text { answered "No" or "Unclear"; if it was } \\
\text { answered "Yes", then the item scored } \\
\text { "1". } \\
\text { Methodological quality will be } \\
\text { considered "low," "moderate," and "high" } \\
\text { if three or less, four to six, and seven to } \\
\text { nine criteria will be met, respectively. }\end{array}$ \\
\hline
\end{tabular}

\section{Data Extraction}

Data will be extracted from papers included in the review using the standardized data extraction tool for prevalence and incidence studies (24) by two independent reviewers. The data extracted will include specific details about the condition, populations, study methods and proportions of interest to the review question and specific objectives including (Annex I):

i. General information: authors/s name, study title and aim, year and country of study, publication type or source of data (journal or report), and socioeconomic data.

ii. Study characteristics: study design, characteristics of the study population (demographic and socioeconomic), sample size, and sampling methods.

iii. Outcome information: proportion of people reported with either current or life time prevalence of domestic violence during diseases outbreaks. 
Any disagreements that arise between the reviewers will be resolved through discussion, or with a third reviewer. Authors of papers will be contacted to request missing or additional data where required.

\section{Data Synthesis}

Papers will, where possible be pooled in statistical meta-analysis using RevMan 5.3 (Copenhagen: The Nordic Cochrane Centre, Cochrane) (25) or any other relevant software. Effect sizes will be expressed as a proportion with $95 \%$ confidence intervals around the summary estimate. Heterogeneity will be assessed statistically using the standard chi-squared, Tau-squared and I-squared tests (26). A random effects model using the double arcsine transformation approach will be used. Subgroup analyses will be conducted where there is sufficient data to investigate disparities in the prevalence of the outcome of interest among different socio demographic and economic status of the study participants. Sensitivity analyses will be conducted to explore the impact of individual studies on the overall calculated prevalence estimates. This will be performed by investigating whether adding or dropping primary studies with slightly unidentified type of domestic violence will make a difference.

Where statistical pooling is not possible due to heterogeneity, the findings will be presented in narrative form including tables and figures to aid in data presentation where appropriate. Source of heterogeneity and reason for which it is determined to be inappropriate to pool data will be specified in the systematic review report.

\section{Discussion}

Now a days our world is facing disastrous events than before, and among these, disease outbreaks be it epidemic or pandemic are the major ones. These disease outbreaks have their own direct impact on the wellbeing of the population in general and more severe impact on the most vulnerable groups, specifically. Beyond a deteriorated health outcome due to the diseases, there is equal or most probably high negative impact on the lives of the poor which will be long lasting and irreversible. We have seen from our last experience that during specific disease epidemics prevalence of domestic violence was almost two fold than the time when there was no epidemics. This study will synthesize an evidence on the prevalence of domestic violence during these catastrophic health events and will provide an input to include violence as part of each assessment. Though we will not include non-English studies, which may cause a publication bias, we will comprehensively retrieve the reference lists of the studies included to obtain more additional studies.

\section{Abbreviations}

COVID-19: Corona Virus and Disease -19

JBI SUMARI: Joanna Briggs Institute - The System for the Unified Management, Assessment and Review of Information 
PRISMA: Preferred Reporting Items for Systematic Reviews and Meta-Analyses

WHO: World Health Organization

\section{Declarations}

\section{Availability of data and materials}

Not applicable.

\section{Ethics approval and consent to participate}

All authors are responsible for all features of this work in confirming that questions related to the accuracy or reliability of any part of the work are appropriately explored and resolved. The present study will not involve any patients and/or the public. No ethical approval or informed consent is required for the purposes of the study.

\section{Consent for publication}

Not applicable.

\section{Availability of data and materials}

Not applicable.

\section{Competing interests}

The authors declare that they have no competing interests

\section{Funding}

None

\section{Authors' contributions}

GA conceptualized the study. GA and BG drafted the manuscript. KY and SM provided edits for the manuscript improvement. GA and KY developed the search strategy. All authors contributed to the revision of the report. Moreover, all authors reviewed the manuscript, approved the final draft, and agreed to submit it for publication. 


\section{Author information}

\section{Affiliations}

Department of health policy and Management, Public Health Faculty, Institute of Health, Jimma University, Jimma, Ethiopia

Gelila Abraham, Kiddus Yitbarek, Beshea Gelana

Department of Health Behaviour and Society, Public Health Faculty, Institute of Health, Jimma University, Jimma, Ethiopia

Sudhakar Morankar

Ethiopian Evidence-Based Healthcare Centre, Health Behaviour and Society Department, Public Health Faculty, Institute of Health, Jimma University, Jimma, Ethiopia

Gelila Abraham, Sudhakar Morankar

\section{References}

1. Wikipedia F encylopedia. Domestic violence [Internet]. [cited 2020 Nov 4]. Available from: https://en.wikipedia.org/wiki/Domestic_violence

2. World Health Organization. Preventing Intinate Partner and Sexual Violence against Women: Taking actiona nad generating evidence. 2010.

3. World health Organization. The W ORLD HEALTH R EPORT 2001. Mental Health: New understanding, new hope [Internet]. 2001. Available from: https://www.who.int/whr/2001/en/whr01_en.pdf?ua=1

4. Michele C. Black, Kathleen C. Basile, Matthew J. Breiding, Sharon G. SmithMikel L. Walters MTMC and MRS. National Intimate Partner and Sexual Violence Survey 2010 Summary Report [Internet]. Atlanta, Georgia: Center for Disease Control and Prevention; 2010. Available from: https://www.cdc.gov/violenceprevention/pdf/nisvs_report2010-a.pdf

5. James F, Anke H. Conflict and Violence Assessement Paper: Benefits and Costs of the Conflict and Violence Targets for the post-2015 Development Agenda. Copenhagen; 2014.

6. Heenan Melanie, Astbury Jill, Vos Theo, Magnus Anne, Piers Leonard S., Walker Lyn, Jenny Morgan $\mathrm{KW}$. The health costs of violence Measuring the burden of disease caused by intimate partner violence [Internet]. Victoria, Australia; 2010. Available from: https://www.vichealth.vic.gov.au/ /media/ResourceCentre/PublicationsandResources/PVAW/IPV BOD web version.ashx

7. World Health Organization. prevalence and health effects of intimate partner violence and nonpartner sexual violence [Internet]. 2013. Available from: 
https://apps.who.int/iris/bitstream/handle/10665/85239/9789241564625_eng.pdf?sequence=1

8. World Health Organization. Violence and disasters [Internet]. 2005. p. 1-2. Available from: https://www.who.int/violence_injury_prevention/publications/violence/violence_disasters.pdf

9. Rezaeian M. Epidemiology of suicide after natural disasters: A review on the literature and a methodological framework for future studies. Am J Disaster Med. 2015;3(September).

10. Onyango MA. Gender-Based Violence Among Adolescent Girls and Young Women: A Neglected Consequence of the West African Ebola Outbreak: Medical , Anthropological , and Public Health Perspectives. 2015.

11. Lisieux E. de Borba Telles, Alexandre M. Valenc,a, Alcina J.S. Barros AG, Silva D. Domestic violence in the COVID-19 pandemic: a forensic psychiatric perspective. Brazillian J Psychatry. 2020;5-6.

12. Parkinson D. Investigating the Increase in Domestic Violence Post Disaster: An Australian Case Study. J Interpers Violence. 2019;(March 2017).

13. Nanthini BS, Nair T. COVID-19 and the Impacts on Women [Internet]. 2020. p. 1-11. Available from: https://reliefweb.int/sites/reliefweb.int/files/resources/NTS-Insight_COVID-19-and-the-Impacts-onWomen-30July2020.pdf

14. Campbell AM. An Increasing Risk of Family Violence during the Covid-19 Pandemic: Strengthening Community Collaborations to Save Lives [Internet]. Forensic Science International: Reports. Elsevier B.V.; 2020. Available from: https://doi.org/10.1016/j.fsir.2020.100089

15. Beland Louis-Philipp, Abel Brodeur, Joanne Haddad DM. COVID-19, Family Stress and Domestic Violence: Remote Work, Isolation and Bargaining Power [Internet]. Bonn, Germany; 2020. Available from: http://ftp.iza.org/dp13332.pdf

16. Campbell AM. An increasing risk of family violence during the Covid-19 pandemic: Strengthening community collaborations to save lives. Forensic Sci Int Reports. 2020;2(January).

17. World Health Organization. Violence - a global public health problem. In: Worl Report on Violence and Health [Internet]. 1999. p. 1-22. Available from:

https://www.who.int/violence_injury_prevention/violence/world_report/en/chap1.pdf?ua=1

18. Shaikh I.A. MA. Emergency preparedness and humanitarian action: the research deficit . Eastern Mediterranean. East Mediterr Heal J. 2006;12:54-63.

19. Juliet Bedford, Jeremy Farra, Chikwe Ihekweazu, Gagandeep Kang, Marion Koopmans and JN. A new twenty-first century science for effective epidemic response. Nature. 2019;575(November).

20. Zachary Munn, Sandeep Moola, Karolina Lisy, Dagmara Riitano CT. Systematic reviews of prevalence and incidence: Checklist for Prevalence Studies. In 2017. p. 7. Available from: https://synthesismanual.jbi.global.

21. David Moher, Larissa Shamseer, Mike Clarke, Davina Ghersi, Alessandro Liberati, Mark Petticrew, Paul Shekelle LAS\& P-PG. Preferred reporting items for systematic review and meta-analysis protocols ( PRISMA-P ) 2015 statement. BMC Syst Rev. 2015;4:1-9. 
22. Joanna Briggs Institute. System for the Unified Management, Assessment, and Review of Information (SUMARI). J Med Libr Assoc. 2019;107(October):634-6.

23. Liberati A, Altman DG, Tetzlaff J, Mulrow C, Gøtzsche PC, John PA. \& reporting The PRISMA statement for reporting systematic reviews and meta-analyses of studies that evaluate healthcare interventions: explanation and elaboration. BMJ. 2009;

24. Zachary Munn, Sandeep Moola, Karolina Lisy, Dagmara Riitano CT. Thank you. 2014.

25. RevMan 5.3 User Guide. 2014. p. 105.

26. Deeks JJ, Altman D. Statistical heterogeneity in systematic reviews of clinical trials: a critical appraisal of guidelines and practice. J Health Serv Res Policy. 2002;7(February).

\section{Appendix}

\section{Appendix I: Data extraction instrument}

\section{JBI Data Extraction form for Prevalence and Incidence Studies}




\section{JBI Data Extraction Form for Prevalence and Incidence Studies}

Study details

Reviewer -

Study ID/Record Number -

Date -

Study title -

Author -

Year -

Journal -

Aims of the study -

Study Method

Setting -

Study design -

Follow-up or study duration -

Subject characteristics -

Dependent variable -

Outcomes -

Outcome measurements -

Ethical approval -

Method of data analysis -

Results

Prevalence n/N (\%)

Proportion and 95\% Confidence Intervals

Incidence $\mathrm{n} / \mathrm{N}(\%)$

Proportion and $95 \%$ Confidence Intervals and duration of recruitment or the study

Authors' comments 


\section{Supplementary Files}

This is a list of supplementary files associated with this preprint. Click to download.

- PRISMAPchecklist.docx

- PRISMAPchecklist.docx 